\title{
Duality, Matroids, Qubits, Twistors, and Surreal Numbers
}

\author{
J. A. Nieto* \\ Facultad de Ciencias Físico-Matemáticas de la Universidad Autónoma de Sinaloa, Culiacán Sinaloa, Mexico
}

We show that via the Grassmann-Plücker relations, the various apparent unrelated concepts, such as duality, matroids, qubits, twistors, and surreal numbers are, in fact, deeply connected. Moreover, we conjecture the possibility that these concepts may be considered as underlying mathematical structures in quantum gravity.

Keywords: duality, matroids, twistors, surreal numbers, quantum gravity

It is a fact that the duality concept is everywhere in both mathematics and physics. Of course, since the list of examples of this fact is very large and since we are concern with quantum gravity let us just briefly mention, as examples in which the duality concept plays a fundamental role, matroid theory [1,2] [see also [3-9] and references therein] and surreal numbers [10-12] in mathematics and string theory [13] and loop quantum gravity [14] in physics. The origin of matroid theory can be traced back to graph theory were according to the Kuratowski theorem a graph has a dual if does not contain the complete graphs $K_{5}$ and $K_{3,3}$ (see [15]). A matroid is a generalization of the graph concept in which every matroid has a dual. One may understand why matroid theory is a generalization of graph theory by associating with every graph $G$ a matroid $M(G)$. So one must have $M\left(K_{5}\right)$ and $M\left(K_{3,3}\right)$, but according to matroid theory one must have the corresponding duals $M^{*}\left(K_{5}\right)$ and $M^{*}\left(K_{3,3}\right)$ which turns out to be non-graphic. A surreal number $x=\left\{X_{L}, X_{R}\right\}$ is written in terms of the dual sets $X_{L}$ left set and $X_{R}$ the right set which satisfies two main axioms (see below). Surprisingly these dual numbers contains the structure of real numbers among other numerical structures. On the other hand it is known that the origin of $M$-theory [16] was inspired by trying to make sense of a number of dualities between string theory and $p$-branes. For instance, in eleven dimensions the 1-brane is dual to the 5-brane (see [16]). Finally, it is known that loop quantum gravity emerges from the discovery of the Ashtekar variables which in turn arises by the requirement of the canonical formalism applied to the self-dual Ricci curvature tensor [see [14] and references therein].

Of course, the duality concepts refereed above may be at first sight quite different for each example. So the first step it is to introduce a formal definition of the concept of duality. It turns out that at least in matroid theory one finds such a formal definition [17]. Let $\mathcal{M}$ denote the family of all matroids $M$ which corresponding to the ground set $E$. The matroid duality is a map * $: \mathcal{M} \rightarrow \mathcal{M}$ satisfying the two main axioms:

$$
\begin{aligned}
(\mathrm{a})^{* *} M & =M & & (\forall M \in \mathcal{M}) . \\
\text { (b) } E\left({ }^{*} M\right) & =E(M) & & (\forall M \in \mathcal{M}) .
\end{aligned}
$$

Inspired by this definition of duality in oriented matroid theory let us propose a general tensor definition of duality structure. Consider a family $\mathcal{A}$ of all completely antisymmetric tensors $A$ ( $p$ forms), which correspond to space of dimension $d$, together with an operation + which can be any well-defined tensorial sum operation. The pair $(\mathcal{A},+)$ determines a dual structure through the map $*: \mathcal{A} \rightarrow \mathcal{A}$ if satisfies the following axioms: 

(I) ${ }^{* *} A=A \quad(\forall A \in \mathcal{A})$.
(II) $d\left({ }^{*} A\right)=d(A) \quad(\forall A \in \mathcal{A})$.

Note that (II) plays the role of (b) in matroid theory.

Assuming the particular case that $\mathcal{A}$ corresponds to family of zero-rank tensors one may add two additional axioms, namely

(III) There exist in $\mathcal{A}$ a self dual element ${ }^{*} 0=0$ such that $A+0=0+A=A, \quad(\forall A \in \mathcal{A})$.

(IV) For $\forall A \in \mathcal{A}$ one has $A+{ }^{*} A={ }^{*} A+A=0$.

One can prove that the element 0 in (III) is unique as follows: Assume that $(\mathcal{A},+)$ is a dual structure with two self-dual elements 0 and $0^{\prime}$. Then $0=0+0^{\prime}=0^{\prime}$. Moreover, according to the axiom (IV) the element ${ }^{*} A$ can be considered as the inverse of $A$. In order to show that the inverse ${ }^{*} A$ is unique one takes recourse of the axiom (I) instead of the associativity axiom in group theory. In fact, assume that an arbitrary element $A$ in $\mathcal{A}$ has two inverses ${ }^{*} A$ and ${ }^{*} B$. Thus, one has (i) $A+{ }^{*} A=0$ and (ii) $A+{ }^{*} B=0$. Applying the axioms (I) and (III) to (ii) one obtains * $A+B=0$ and therefore according (i) one gets ${ }^{*} A+B={ }^{*} A+A$ which means that $B=A$. The two axioms (III) and (IV) are similar to the definition of a field in number theory. For these reasons one it is straightforward to verify that the integer $Z$ and the real numbers $R$ are in fact dual structures.

The main goal of the present work is to comment about the possibility that the various concepts such as oriented matroids, qubits, twistors, and surreal numbers are linked by the duality symmetry. Moreover we shall argue that such a dual concept may be considered as an underlying mathematical tool in quantum gravity.

It turns out that the completely antisymmetric $\varepsilon$-symbol becomes the underlying mathematical object in all these connections. Specifically, the $\varepsilon$-symbol can be defined as

$$
\varepsilon^{a_{1} \ldots a_{d}} \in\{-1,0,1\}
$$

Here, the indices $a_{1}, \ldots, a_{d}$ run from 1 to $d$. This is a $d$-rank density tensor which values are +1 or -1 depending on even or odd permutations of $\varepsilon^{12 \ldots d}$, respectively. Moreover, $\varepsilon^{a_{1} \ldots a_{d}}$ takes the value 0 unless the values of $a_{1} \ldots a_{d}$ are all different. Lowering and rising the indices with a Kronecker delta $\delta_{a b}$ one finds that

$$
\varepsilon^{a_{1} \ldots a_{d}} \varepsilon_{b_{1} \ldots b_{d}}=\delta_{b_{1} \ldots b_{d}}^{a_{1} \ldots a_{d}}
$$

where $\delta_{b_{1} \ldots b_{d}}^{a_{1} \ldots a_{d}}$ is a generalized Kronecker delta. A contraction in (2) of the last $n$-indices of the type $a_{i}$ with the last $n$-indices of the type $b_{i}$ leads to

$$
\varepsilon^{a_{1} \ldots a_{k-1} a_{k} \ldots a_{d}} \varepsilon_{b_{1} \ldots b_{k-1} a_{k} \ldots a_{d}}=n ! \delta_{b_{1} \ldots b_{k-1}}^{a_{1} \ldots a_{k-1}},
$$

with $n=d-k+1$. In particular one has

$$
\varepsilon^{a_{1} \ldots a_{d}} \varepsilon_{a_{1} \ldots a_{d}}=d !
$$

Let $v_{a}^{i}$ be any $d \times n$ matrix over some field $F$, where the index $i$ takes values in the ordinal set $E=\{1, \ldots, n\}$. Consider the object

$$
\Sigma^{i_{1} \ldots i_{d}}=\varepsilon^{a_{1} \ldots a_{d}} v_{a_{1}}^{i_{1}} \ldots v_{a_{d}}^{i_{d}} .
$$

Using the $\varepsilon$-symbol property

$$
\varepsilon^{a_{1} \ldots\left[a_{d}\right.} \varepsilon^{\left.b_{1} \ldots b_{d}\right]}=0
$$

it is not difficult to prove that $\Sigma^{i_{1} \ldots i_{d}}$ satisfies the GrassmannPlücker relations [see [18] and references therein], namely

$$
\Sigma^{i_{1} \ldots\left[i_{d}\right.} \Sigma^{\left.j_{1} \ldots j_{d}\right]}=0 .
$$

Here, the brackets in the indices of (6) and (7) mean completely antisymmetric.

Through (5) one can define the object

$$
\Sigma=\frac{1}{d !} \Sigma^{i_{1} \ldots i_{d}} e_{i_{1}} \wedge e_{i_{2}} \wedge \ldots \wedge e_{i_{d}}
$$

where $e_{i_{1}}, e_{i_{2}}, \ldots, e_{i_{d}}$ are 1 -form bases associated with the $\left(\begin{array}{l}n \\ d\end{array}\right)$ dimensional real vector space of alternating $d$-forms on $R^{n}$. It turns out that (8) can also be written as

$$
\Sigma=\mathbf{v}_{1} \wedge \mathbf{v}_{2} \wedge \ldots \wedge \mathbf{v}_{d}
$$

for some $\mathbf{v}_{1}, \mathbf{v}_{2}, \ldots, \mathbf{v}_{d} \in R^{n}$. This means that $\Sigma^{i_{1} \ldots i_{d}}$ corresponds to an alternating decomposable $d$-form [19].

A realizable chirotope $\chi$ is defined as

$$
\chi^{i_{1} \ldots i_{d}}=\operatorname{sign} \Sigma^{i_{1} \ldots i_{d}} .
$$

In order to define non-realizable chirotopes it is convenient to write the expression (7) in the alternative form

$$
\sum_{k=1}^{d+1} s_{k}=0,
$$

where

$$
s_{k}=(-1)^{k} \Sigma^{i_{1} \ldots i_{d-1} j_{k}} \Sigma^{j_{1} \ldots \hat{j}_{k} \cdots j_{d+1}} .
$$

Here, $j_{d+1}=i_{d}$ and $\hat{\jmath}_{k}$ establish the notation for omitting this index. Thus, for a general definition, one defines a $d$-rank chirotope $\chi: E^{d} \rightarrow\{-1,0,1\}$ if there exist $r_{1}, \ldots, r_{d+1} \in R^{+}$such that

$$
\sum_{k=1}^{d+1} r_{k} s_{k}=0
$$

with

$$
s_{k}=(-1)^{k} \chi^{i_{1} \ldots i_{d-1} j_{k}} \chi^{j_{1} \ldots \hat{j}_{k} \ldots j_{d+1}},
$$


and $k=1, \ldots, d+1$. It is evident that $(11)$ is a particular case of (13). Therefore, there are chirotopes that may be non-realizable. Moreover, this definition of a chirotope admits a straightforward generalization to the complex structure setting. In this case the complex chirotopes are called phirotopes [20-22].

Given a chirotope (or phirotope) $\chi^{i_{1} \ldots i_{d}}$ its dual is defined as

$$
{ }^{*} \chi_{i_{d+1} \ldots i_{p}}=\varepsilon_{i_{1} \ldots i_{d} i_{d+1} \ldots i_{p}} \chi^{i_{1} \ldots i_{d}} .
$$

Here $D=d+p$ is the total dimension of the ground state $E$. Observe that due to the relations (3) one gets

$$
{ }^{* *} \chi=\chi
$$

which means that $\chi$ satisfies the axiom (I). It turns out that (16) is true for a general completely antisymmetric object $F$ ( $d$-form) when its dual is defined in terms of the $\varepsilon$-symbol. In fact, when $D$ is even one can write $D=d+d=2 d$ and in this case one can define the self-dual (antiself-dual) tensor as

$$
{ }^{ \pm} F=F \pm{ }^{*} F
$$

One observe that ${ }^{ \pm} F$ satisfies

$$
{ }^{* \pm} F= \pm{ }^{ \pm} F
$$

Thus, one sees that for $D$ even the ${ }^{ \pm} F$ tensor is another self-dual (antiself-dual) notion other than the 0 element in the axioms (III) and (IV).

Let us now explain how the Grassmann-Plücker relation (7) is connected with qubit theory [see [23] and references therein]. For this purpose consider the general complex state $\mid \psi>\in C^{2^{N}}$

$$
\left|\psi>=\sum_{A_{1}, A_{2}, \ldots, A_{N}=0}^{1} Q_{A_{1} A_{2} \ldots A_{N}}\right| A_{1} A_{2} \ldots A_{N}>
$$

where the states $\left|A_{1} A_{2} \ldots A_{N}>=\right| A_{1}>\otimes\left|A_{2}>\ldots \otimes\right| A_{N}>$ correspond to a standard basis of the $N$-qubit. For a 3-qubit (19) becomes

$$
\left|\psi>=\sum_{A_{1}, A_{2}, A_{3}=0}^{1} Q_{A_{1} A_{2} A_{3}}\right| A_{1} A_{2} A_{3}>
$$

while for 4-qubit one has

$$
\left|\psi>=\sum_{A_{1}, A_{2}, A_{3}, A_{4}=0}^{1} Q_{A_{1} A_{2} A_{3} A_{4}}\right| A_{1} A_{2} A_{3} A_{4}>.
$$

It turns out that, in a particular subclass of $N$-qubit entanglement, the Hilbert space can be broken into the form $C^{2^{N}}=C^{L} \otimes C^{l}$, with $L=2^{N-n}$ and $l=2^{n}$. Such a partition allows a geometric interpretation in terms of the complex Grassmannian variety
$G r(L, l)$ of $l$-planes in $C^{L}$ via the Plücker embedding. It turns out that in this scenario the complex 3-qubit, 4-qubit admit a geometric interpretation in terms of the complex Grassmannian varieties $\operatorname{Gr}(4,2), \operatorname{Gr}(8,2)$, respectively [see [23] for details]. The idea is to associate the first $N-n$ and the last $n$ indices of $Q_{A_{1} A_{2} \ldots A_{N}}$ with a $L \times l$ matrix $\omega_{a_{1}}^{i_{1}}$. This can be interpreted as the coordinates of the Grassmannian $G r(L, l)$ of $l$-planes in $C^{L}$. Using the matrix $\omega_{p_{1}}^{i_{1}}$ one can define the Plücker coordinates

$$
\mathcal{Q}^{i_{1} \ldots i_{d}}=\varepsilon^{a_{1} \ldots a_{d}} \omega_{a_{1}}^{i_{1}} \ldots \omega_{a_{d}}^{i_{d}},
$$

which one recognizes as the complex version of the decomposable tensor $\Sigma^{i_{1} \ldots i_{d}}$ defined in (5). Moreover, one verifies that under the transformation $\omega \rightarrow S \omega$ with $S \in G L(l, C)$ the Plücker coordinates transform as $\mathcal{Q}^{i_{1} \ldots i_{d}} \rightarrow \operatorname{Det}(S) \mathcal{Q}^{i_{1} \ldots i_{d}}$ and of course $\pm^{i_{1} \ldots i_{d}}$ satisfies the Grassmann-Plücker relations

$$
\mathcal{Q}^{i_{1} \ldots\left[i_{d}\right.} \mathcal{Q}^{\left.j_{1} \ldots j_{d}\right]}=0 .
$$

Now, consider the quantity $\sigma_{\mu}=\left(\sigma_{0}, \sigma_{\hat{\imath}}\right)$, where the $\sigma_{\hat{\imath}}$ denotes Pauli matrices and $\sigma_{0}$ is the identity matrix. By using $\sigma_{\mu}$ the linear momentum in 4-dimensions $p^{\mu}$ can be written as

$$
p^{A \dot{B}}=\sigma_{\mu}^{A \dot{B}} p^{\mu}
$$

This is the spinorial representation of $p^{\mu}$. An interesting aspect emerges if one sets $\operatorname{Det}\left(p^{A \dot{B}}\right)=0$, corresponding to a null momentum $p^{\mu} p_{\mu}=0$. This means that

$$
\frac{1}{2 !} \varepsilon_{A C} \varepsilon_{\dot{B} \dot{D}} p^{A \dot{B}} p^{C \dot{D}}=0
$$

A solution to this equation is given by $p^{A \dot{B}}=\xi^{A} \eta^{\dot{B}}$. Since $p^{\mu}$ is real vector one verifies that $p^{A \dot{B}}=\bar{p}^{\dot{B} A}$ and therefore

$$
\xi^{A} \eta^{\dot{B}}=\bar{\xi}^{\dot{B}} \bar{\eta}^{A}
$$

One finds that this last expression means that $\eta^{\dot{B}}=a \bar{\xi}^{\dot{B}}$, where due to (26) one sees that $a$ is real number which can be normalized in the form $a= \pm$. So one has found that, in the case of null momentum, one can write $p^{A \dot{B}}$ in terms of a more fundamental complex quantity $\xi^{A}$, namely

$$
p^{A \dot{B}}= \pm \xi^{A} \bar{\xi}^{\dot{B}}
$$

Similar analysis applies to the total angular momentum $M^{\mu \nu}=$ $x^{\mu} p^{v}-x^{v} p^{\mu}+S^{\mu \nu}$, where $S^{\mu \nu}$ is the internal angular momentum satisfying the Tulczyjew second class constraint [24];

$$
S^{\mu v} p_{v}=0
$$

Observe that due to (28) and since $p^{\mu}$ is a null vector one has $M^{\mu v} p_{v}=-\left(x^{v} p_{v}\right) p^{\mu}$ This means that $\delta_{\alpha \beta \gamma}^{\tau \mu \nu} p^{\alpha} M^{\beta \gamma} p_{v}=$ 0 . In turn this leads to $\varepsilon_{\sigma \alpha \beta \gamma} \varepsilon^{\sigma \tau \mu \nu} p^{\alpha} M^{\beta \gamma} p_{v}=0$ or 
$\varepsilon_{\sigma \alpha \beta \gamma} \varepsilon^{\sigma \tau \mu \nu} p^{\alpha} S^{\beta \gamma} p_{v}=0$. Therefore, if one defines the 4vector $S_{\sigma}=\frac{1}{2} \varepsilon_{\sigma \alpha \beta \gamma} p^{\alpha} S^{\beta \gamma}$ one obtains $\varepsilon^{\sigma \tau \mu \nu} S_{\sigma} p_{\nu}=0$ and consequently one discovers that

$$
S_{\mu}=s p_{\mu}
$$

for some non-vanishing constant $s$ which is identified with the helicity of the system. This means that the spin $S_{\mu}$ is parallel or anti-parallel to $p_{\mu}$ depending of the sign of $s$. So, determining $p^{A \dot{B}}$ in terms of $\xi^{A}$ via (27) is equivalent to determine $S^{A \dot{B}}$ in the form $S^{A \dot{B}}=s \xi^{A} \bar{\xi}^{\dot{B}}$. Thus, considering (28) one sees that the left relevant part of $M^{\mu \nu}$ is

$$
L^{\mu \nu}=x^{\mu} p^{\nu}-x^{\nu} p^{\mu},
$$

but again since $p^{\mu}$ is a null vector one has $L^{\mu v} p_{v}=-\left(x^{v} p_{v}\right) p^{\mu}$ which means that out of the six true degrees of freedom of $L^{\mu \nu}=-L^{\nu \mu}$ three are already determined by $p^{\mu}$. Therefore, the corresponding spinor representation of $L^{\mu \nu}$, namely $L^{A \dot{B} C \dot{D}}=$ $\sigma_{\mu}^{A \dot{B}} \sigma_{\nu}^{C \dot{D}} L^{\mu \nu}$, can be written as

$$
L^{A \dot{B} C \dot{D}}=\mu^{A C} \epsilon^{\dot{B} \dot{D}}+\epsilon^{A C} \mu^{\dot{B} \dot{D}} .
$$

Here, $\mu^{A C}=\mu^{C A}$ is a symmetric matrix and therefore has only three independent complex degrees of freedom. In order to reduce $\mu^{A C}$ to only three real components which of course are related to the true three degrees of freedom of $L^{\mu \nu}$ one further writes $\mu^{A C}$ in the form $\mu^{A C}=\xi^{A} \pi^{C}+\xi^{C} \pi^{A}$. If to the coordinates $\xi_{\dot{A}}$ one adds the the spinor $\pi^{A}$ one is lead to the twistor structure $\mathcal{P}^{\alpha}=\left(\pi^{A}, \xi_{\dot{A}}\right)$ [25] [see [26] and references therein] which can be identified with a point in $C^{4}$. This analysis revel that in the case of a null system the eight coordinates $\left(x^{\mu}, p^{\nu}\right)$ in $R^{8}$ may in principle be associated with the coordinates $\left(\pi^{A}, \xi_{\dot{A}}\right)$ in the twistor complex space $C^{4}$.

Consider the 2-index twistor

$$
P^{\alpha \beta}=\mathcal{P}_{1}^{\alpha} \mathcal{P}_{2}^{\beta}-\mathcal{P}_{2}^{\alpha} \mathcal{P}_{1}^{\beta},
$$

which can also be written as

$$
\mathcal{P}^{\alpha \beta}=\varepsilon^{a b} \mathcal{P}_{a}^{\alpha} \mathcal{P}_{b}^{\beta} .
$$

If one defines $p_{1}^{\mu}=x^{\mu}$ and $p_{2}^{\mu}=p^{\mu}$ one sees that $L^{\mu \nu}$ can be written as

$$
L^{\mu \nu}=\varepsilon^{a b} p_{a}^{\mu} p_{b}^{v}
$$

and therefore one concludes that $\mathcal{P}^{\alpha \beta}$ can be understood as the complexification of $L^{\mu \nu}$. Of course, $\mathcal{P}^{\alpha \beta}$ satisfies the GrassmannPlücker relations

$$
\mathcal{P}^{\mu[v} \mathcal{P}^{\alpha \beta]}=0
$$

It turns out that $\mathcal{P}^{\alpha \beta}$ can be used to associate points in $C^{4}$ with points in the complexified Minkowski space (see [25]).
From the perspective of oriented complex matroids, $\mathcal{P}^{\alpha \beta}$ is just a representable phirotope. One is tempted to assume that a generalization of twistor theory may be also be associated with the phirotope theory.

Is it possible that twistors or qubits can be related to surreal number theory [10-12]? Consider the set

$$
x=\left\{X_{L} \mid X_{R}\right\}
$$

and call $X_{L}$ and $X_{R}$ the left and right sets of $x$, respectively. Conway develops the surreal numbers structure $\mathcal{S}$ from two axioms:

Axiom 1. Every surreal number corresponds to two sets $X_{L}$ and $X_{R}$ of previously created numbers, such that no member of the left set $x_{L} \in X_{L}$ is greater or equal to any member $x_{R}$ of the right set $X_{R}$.

Let us denote by the symbol $\Varangle$ the notion of no greater or equal to. So the axiom establishes that if $x$ is a surreal number then for each $x_{L} \in X_{L}$ and $x_{R} \in X_{R}$ one has $x_{L} \nsucceq x_{R}$. This is denoted by $X_{L} \ngtr X_{R}$.

Axiom 2. One number $x=\left\{X_{L} \mid X_{R}\right\}$ is less than or equal to another number $y=\left\{Y_{L} \mid Y_{R}\right\}$ if and only the two conditions $X_{L} \ngtr y$ and $x \nsupseteq Y_{R}$ are satisfied.

This can be simplified by saying that $x \leq y$ if and only if $X_{L} \ngtr y$ and $x \nsupseteq Y_{R}$.

Observe that Conway definition relies in an inductive method; before a surreal number $x$ is introduced one needs to know the two sets $X_{L}$ and $X_{R}$ of surreal numbers. Using Conway algorithm one finds that at the $j$-day one obtains $2^{j+1}-1$ numbers all of which are of form

$$
x=\frac{m}{2^{n}},
$$

where $m$ is an integer and $n$ is a natural number, $n>0$. Of course, the numbers (37) are dyadic rationals which are dense in the reals $R$.

The sum and product of surreal numbers are defined as

$$
x+y=\left\{X_{L}+y, x+Y_{L} \mid X_{R}+y, x+Y_{R}\right\}
$$

and

$$
\begin{gathered}
x y=\left\{X_{L} y+x Y_{L}-X_{L} Y_{L}, X_{R} y+x Y_{R}-X_{R} Y_{R} \mid X_{L} y+x Y_{R}\right. \\
\left.-X_{L} Y_{R}, X_{R} y+x Y_{L}-X_{R} Y_{L}\right\}
\end{gathered}
$$

respectively. The importance of (38) and (39) is that allow us to prove that the surreal number structure is algebraically a closed field. Moreover, through (38) and (39) it is also possible to show 
that the real numbers $R$ are contained in the surreals $\mathcal{S}$ [see [1012] for details]. Of course, in some sense the prove relies on the fact that the dyadic numbers (37) are dense in the reals $R$.

In 1986, Gonshor [12] introduced a different but equivalent definition of surreal numbers.

Definition 1. A surreal number is a function $f$ from initial segment of the ordinals into the set $\{+,-\}$.

For instance, if $f$ is the function so that $f(1)=+, f(2)=+$, $f(3)=-, f(4)=+$ then $f$ is the surreal number $(++-+)$. In the Gonshor approach one obtains the sequence: 1-day

$$
-1=(-), \quad(+)=+1,
$$

in the 2-day

$$
-2=(--), \quad-\frac{1}{2}=(-+), \quad(+-)=+\frac{1}{2}, \quad(++)=+2,
$$

and 3-day

$$
\begin{aligned}
& -3=(---), \quad-\frac{3}{2}=(--+), \quad-\frac{3}{4}=(-+-), \\
& -\frac{1}{4}=(-++),(+--)=+\frac{1}{4}, \quad(+-+)=+\frac{3}{4}, \\
& (++-)=+\frac{3}{2}, \quad(+++)=+3,
\end{aligned}
$$

respectively. Moreover, in Gonshor approach one finds the different numbers through the formula

$$
\mathcal{J}=l\left|\varepsilon_{0}\right|-\frac{\left|\varepsilon_{1}\right|}{2}+\sum_{i=2}^{s} \frac{\left|\varepsilon_{i}\right|}{2^{i}}
$$

where $\varepsilon_{0}, \varepsilon_{1}, \varepsilon_{2}, \ldots, \varepsilon_{q} \in\{+,-\}$ and $\varepsilon_{0} \neq \varepsilon_{1}$. Furthermore, one has $|+|=+$ and $|-|=-$. As in the case of Conway definition, through (43) one gets the dyadic rationals. Just for clarity, let us consider the additional example:

$$
(++-+-+)=2-\frac{1}{2}+\frac{1}{4}-\frac{1}{8}+\frac{1}{16}=\frac{27}{16} .
$$

By defining the order $x<y$ if $x(\alpha)<y(\alpha)$, where $\alpha$ is the first place where $x$ and $y$ differ and the convention $-<0<+$, it is possible to show that the Conway and Gonshor definitions of surreal numbers are equivalent [see [12] for details].

Suppose that instead of qubits we consider a rebit (real bits) which can be thought as $j$-tensor [4],

$$
t_{A_{1} A_{2} \ldots A_{j}}
$$

where the indices $A_{1}, A_{2}, \ldots, A_{j}$ run from 0 to 1 . Of course $j$ indicates the rank of $t_{A_{1} A_{2} \ldots A_{j}}$. In tensorial analysis, (45) is a familiar object. One arrives to a link with surreal numbers by making the indices identification $0 \rightarrow+$ and $1 \rightarrow-$. For instance, the tensor $t_{0010}$ in the Gonshor notation becomes

$$
t_{0010} \rightarrow t_{++-+} \rightarrow(++-+)
$$

In terms of $t_{A_{1} A_{2} \ldots A_{j}}$, the expressions (40), (41) and (42) read

$$
-1=t_{1}, \quad t_{0}=+1,
$$

in the 2-day

$$
-2=t_{11}, \quad-\frac{1}{2}=t_{10}, \quad t_{01}=\frac{1}{2}, \quad t_{00}=2,
$$

and 3-day

$$
\begin{gathered}
-3=t_{111}, \quad-\frac{3}{2}=t_{110}, \quad-\frac{3}{4}=t_{101}, \quad-\frac{1}{4}=t_{100}, \\
t_{011}=+\frac{1}{4}, \quad t_{010}=+\frac{3}{4}, \quad t_{001}=+\frac{3}{2}, \quad t_{000}=+3,
\end{gathered}
$$

respectively.

Note that there is a duality symmetry between positive and negative labels in surreal numbers. In fact, one can prove that this is general for any $j$-day. This could be anticipated because according to Conway definition a surreal number can be written in terms of the dual pair left and right sets $X_{L}$ and $X_{R}$. Further, the concept of duality it is even clearer in the Gonshor definition of surreal numbers since in such a case one has a functions $f$ with the image in the dual set $\{+,-\}$. In terms of the tensor $t_{A_{1} A_{2} \ldots A_{p}}$ such a duality can be written in the form

$$
t_{A_{1} A_{2} \ldots A_{p}}+(-1)^{p} \varepsilon_{A_{1} B_{1}} \varepsilon_{A_{2} B_{2} \ldots \varepsilon_{A_{p} B_{p}}} t^{B_{1} B_{2} \ldots B_{p}}=0,
$$

where

$$
\varepsilon_{A B}=\left(\begin{array}{cc}
0 & 1 \\
-1 & 0
\end{array}\right)
$$

The identification of surreal numbers with rebits means that its complexification must be related to qubit theory and therefore with twistor theory. So one has discovered that the use of the completely antisymmetric object epsilon $\varepsilon^{a_{1} \ldots a_{d}}$ allows to define the Plucker coordinates which must to satisfy the GrassmannPlücker relation. In turn, we have proved that this relation is a common mathematical central notion in oriented matroids, qubit theoy, twistor theory and surreal number theory.

Moreover, it has been proved in Mosseri and Dandoloff [27], Mosseri [28], and Bernevig and Chen [29] that for normalized qubits the complex 1-qubit, 2-qubit, and 3-qubit are deeply related to division algebras via the Hopf maps, $S^{3} \stackrel{S^{1}}{\longrightarrow} S^{2}, S^{7} \stackrel{S^{3}}{\longrightarrow}$ $S^{4}$, and $S^{15} \stackrel{S^{7}}{\longrightarrow} S^{8}$, respectively. It seems that there does not exist a Hopf map for higher $N$-qubit states. So, from the perspective of Hopf maps, and therefore of division algebras, one arrives 
to the conclusion that 1-qubit, 2-qubit, and 3-qubit are more special than higher dimensional qubits [see [27-29] for details]. Again one wonders whether surreal numbers can contribute in this qubits theory framework.

The original idea of Penrose was to replace the continuity of the Minkowski space for new geometric framework which may allow for a discrete structure and in this way unify general relativity and quantum mechanics. In fact, one of the original motivation to introduce twistors was be able to have mathematical arena in which the discretization of the spacetime was possible. The hope was that the complex structure of twistors may be connected with quantum mechanics. In a sense the idea was to replace $R^{4}$ by $C^{4}$ and in this way, since the object in $C^{4}$ are complex, one may be able to connect with quantum mechanics which intrinsically is a complex structure. Ironically, according to the discussion in this work, it seems to us that the combinatorial structure searched by Penrose in connection with quantum gravity is not the twistors itself but the underlying oriented matroid theory. But ground set in oriented matroids can be constructed by strings of the set $\{+-\}$ which are the main tool in qubit theory and surreal numbers. All these comments suggested that the concepts such as chirotopes (phirotopes), qubits, twistors, and surreals must be considered mathematical tools underlying quantum gravity.

Let us analysis deeply the connection between surreal numbers and qubits. For this purpose we shall assume that one may be able to write a surreal complex numbers $\mathcal{Z}$ in the form

$$
\mathcal{Z}=\mathcal{J}_{1}+i \mathcal{J}_{2}
$$

where $\mathcal{J}_{1}$ and $\mathcal{J}_{2}$ are two surreal numbers according to the formula (43). This complexification of surreal numbers must establish a complete connection with the $N$-qubit structure if one assume the existence of a complex operator $\hat{\mathcal{Z}}_{A_{1} A_{2} \ldots A_{N}}$ such that

$$
\begin{aligned}
\hat{\mathcal{Z}}_{A_{1} A_{2} \ldots A_{N}} \mid A_{1} A_{2} \ldots A_{N}> & =\sum_{A_{1}, A_{2}, \ldots, A_{N}=0}^{1} \\
Q_{A_{1} A_{2} \ldots A_{N}} \mid A_{1} A_{2} \ldots A_{N}> & =\mathcal{J} \mid A_{1} A_{2} \ldots A_{N}>.
\end{aligned}
$$

This is inspired in the observation that $\mathcal{J}$ in (43) can be associated with the eigenvalues of a $z$-component $\hat{J}_{z}$ of the total angular momentum $\hat{J}$ in quantum mechanics. Of course in such case one has $J_{z}=l \pm \frac{1}{2}$, with the identification of $\frac{1}{2}$-spin of the system. The surprise with surreal numbers is that predicts that besides $\frac{1}{2}$-spin system there must exist infinite number of $\mathcal{J}$-spins, according to the formula (43). Thus, for instance one must include particles with $\frac{1}{4}$-spin (see $\left.[30,31]\right)$ and $\frac{1}{8}$-spin and in general particles with dyadic rational $\frac{m}{2^{n}}$-spin.

Traditionally, quantum mechanics enter in the above twistor formalism when one writes all possible gauge fields (and their associated field equations) in twistor language and proceed to quantize in the usual way. In the case of qubit theory things are different because, even from the begining, qubits refers to quantum states. Thus, concepts of quantum mechanics such as the density of states are constructed from the corresponding entanglement monotones [23]. Here, we would like to propose an alternative possible route to connect further our formalism with quantum mechanics. The central idea is to continue looking the surreal numbers as a quantities associated with different dyadic spins $\left(\frac{m}{2^{n}}\right.$-spin). Let us explain in some detail this idea. As we mentioned $\mathcal{J}$ in (43) seems to play the analog of the eigenvalues of the $z$-component $\hat{J}_{z}$ of the angular momentum operator, namely $J_{z}=l \pm \frac{1}{2}$. Roughly speaking, from the point of view of number theory, the quantization of a physical system means to go from the real numbers (continuum) $R$ to natural numbers $N$ (discrete). In the case of surreal numbers things are different because one starts with the 0-day, 1-day, 2-day, and so on and in the $\omega$-day (this is the way mathematitians called) one obtains the real numbers $R$. In other words one starts with a discrete structure and finds the continuum scenario. Moreover, if in addition to (43) one uses the identity

$$
2^{n+1}=2+2+4+8+\ldots+2^{n},
$$

it is not difficult to show that $\mathcal{J}$ in (43) satisfies the expression

$$
-l<\mathcal{J}<l
$$

Since $l<j$ one also has

$$
-j<\mathcal{J}<j
$$

Here, one assumes that from (43) one has $j=l+s$. Of course, (56) is the analoguos inequality of the eigenvalue of the total angular momentum. Following this route of thoughts one first note that surreal numbers of the type $(++\ldots++$ ) (or the corresponding negative part) can be associated with higher integer-spins, $1,2,3, \ldots$, while surreal numbers of the type $(++$ $\ldots+-)$ can be associated with half-inter spins, $1 / 2,3 / 2,5 / 2, \ldots$. This means that in principle bosons and fermions are part of the surreal structure and therefore supersymmetry must be present. Thus one must expect that a generalized supersymmetry can be obtained if one includes other surreal numbers such as $1 / 4,3 / 4,1 / 8,3 / 8$, and so on. Since, as we mentioned, the dyadic rational $m / 2^{n}$ are dense in the reals $R$ one should expect that eventually, in the $\omega$-day, the anyons may emerge. What about the graviton? This corresponds to the surreal number 2 or 2 spin. Thus, just as in string theory the graviton is just one resonance out of many or even infinity resonances, in our case the graviton is just a physical system with particular value 2-spin, but in principle one has all kind of dyadic-spin particles. Thus, according to these observations it seems that quantum gravity should not be seen as an isolated problem but as part of a much larger system in which all types of dyadic-spins are present.

Another source of interesting developments it may emerge from the analysis of singularities, both in balck-holes and cosmology. In fact, from the point of view of surreal numbers theory the black-hole singularity $2 M G / c^{2} r \rightarrow \infty$, when $r \rightarrow 0$, and the Big-Bang singularity (of the radiation energy density) $\rho_{r}=\rho_{0} / a^{4} \rightarrow \infty$, when $a \rightarrow 0$ are not a real problem because in such a mathematical theory all kind of infinite large and infinite small are present. 
It is worth mentioning that in Atiyah [32], the twistor space and the Plücker coordinates are used to determine the geometry of the instantons solutions of Yang-Mills theory. It may interesting for further research to find the connection between instantons formalism and surreal number theory.

Finally, let us just mention that using fiber bundle concept in oriented matroid theory $[33,34]$ a connection with $p$-branes and phirotopes was established [6]. Thus according to the present development one may expect that eventually a link between $p$ branes and surreal numbers must be route to follow in the quest of quantum gravity.

\section{REFERENCES}

1. Oxley JG. Matroid Theory. New York, NY: Oxford Science Publications; Oxford University Press (1992).

2. Björner A, Las Vergnas M, Sturmfels B, White N, Ziegler GM. Oriented Matroids. Cambridge: Cambridge University Press (1993).

3. Nieto JA. Matroids and p-branes. Adv Theor Math Phys. (2004) 8:177-88. doi: 10.4310/ATMP.2004.v8.n1.a4

4. Nieto JA. Oriented matroid theory as a mathematical framework for M-theory Adv Theor Math Phys. (2006) 10:747-57. doi: 10.4310/ATMP.2006.v10.n5.a5

5. Nieto JA. Searching for a connection between matroid theory and string theory. J Math Phys. (2004) 45:285. doi: 10.1063/1.1625416

6. Nieto JA. Phirotopes, super p-branes and Qubit theory. Nucl Phys B (2014) 883:350-72. doi: 10.1016/j.nuclphysb.2014.04.001

7. Nieto JA, Marin MC. Matroid theory and Chern-Simons. J Math Phys. (2000) 41:7997-8005. doi: 10.1063/1.1319518

8. Nieto JA. Qubits and oriented matroids in four time and four space dimensions. Phys Lett B (2013) 718:1543-47. doi: 10.1016/j.physletb.2012.12. 034

9. Nieto JA. Qubits and chirotopes. Phys Lett B (2010) 692:43-6. doi: 10.1016/j.physletb.2010.07.010

10. Knuth DE. Surreal Numbers: How Two Ex-Students Turned on to Pure Mathematics and Found Total Happiness: A Mathematical Novelette. Boston, MA: Addison -Wesley Publising Co. (1974).

11. Conway JH. On Number and Games. London: Academic Press (1976).

12. Gonshor H. An Introduction to the Theory of Surreal Numbers. Cambridge: Cambridge University Press (1986).

13. Green M, Schwarz J, Witten E. Superstring Theory. Cambridge, UK: Cambridge University Press (1987).

14. Ashtekar A, Lewandowski J. Background independent quantum gravity: a status report. Class Quant Grav. (2004) 21:R53. doi: 10.1088/0264-9381/21/15/R01

15. Whitney H. On the abstract properties of linear dependence. Am J Math. (1935) 57:509-33.

16. Duff MJ. M-theory (the theory formerly known as strings). Int J Mod Phys A (1996) 11:5623-42.

17. Bland RG, Dietrich BL. A unified interpretation of several combinatorial dualities. Discrete Optim.(2008) 5:337-49. doi: 10.1016/j.disopt.2006.08.002

18. Bokowski J, Sturmfels B.Computational Synthetic Geometry. Berlin; Heidelberg; New York, NY: Spring-Verlag (1980).

19. Nash C, Sen S. Topology and Geometry for Physicists. London: Academic Press (1983).

20. Below A, Krummeck V, Richter-Gebert J. Complex matroids phirotopes and their realizations in Rank 2. In: Aronov B, Basu S, Pach J, Sharir M, editors.

\section{AUTHOR CONTRIBUTIONS}

The author confirms being the sole contributor of this work and has approved it for publication.

\section{ACKNOWLEDGMENTS}

I would like to thank the Mathematical, Computational \& Modeling Sciences Center of the Arizona State University where part of this work was developed. I would like also to thank the two referees and the editor for valuable comments.

Discrete and Computational Geometry. Algorithms and Combinatorics, Vol. 25. Berlin; Heidelberg: Springer (2003). p. 203-33.

21. Ziegler G, Gunter M. What is a complex matroid? Dis. Comput. Geom.(1993) 10:313-48.

22. Delucchi E. On Generalizing Oriented Matroids to a Complex Setting. Thesis, ETH Zurich.

23. Levay P. On the geometry of a class of $\mathrm{N}$-qubit entanglement monotones. $J$ Phys A (2005) 38:9075-85. doi: 10.1088/0305-4470/38/41/016

24. Tulczyjew W. Motion of multipole particles in general relativity theory. Acta Phys Pol. (1959) 18:393-408.

25. Penrose R, MacCallum MAH. Twistor theory: an approach to the quantisation of fields and space-time. Phys Rep. (1972) 6:241-315. 241.

26. Ward S, Wells RO. Twistor Geometry and Field Theory. Cambridge: Cambridge University Press (1991).

27. Mosseri R, Dandoloff R. Geometry of entangled states, Bloch spheres and Hopf fibrations. J Phys A (2001) 34:10243. doi: 10.1088/0305-4470/34/47/324

28. Mosseri R. Two-qubit and three-qubit geometry and Hopf fibrations. In: Monastyrsky MI, editors. Topology in Condensed Matter. Vol 150. Berlin: Heidelberg: Springer (2006). p. 187-203.

29. Bernevig BA, Chen HD. Geometry of the three-qubit state, entanglement and division algebras. J Phys A (2003) 36:8325. doi: 10.1088/0305-4470/36/30/309

30. Mezincescu L, Townsend PK. Anyons from strings. Phys Rev Lett. (2010) 105:191601. doi: 10.1103/PhysRevLett.105.191601

31. Soroking D. The Heisenberg Algebra and Spin. Fortsch. Phys. (2002) 50: 724 doi: 10.1002/1521-3978(200205)50:5/7<724::AID-PROP724>3.0.CO;2-J

32. Atiyah MF. Geometry of Yang-Mills fields, Scuola Normale Superiore. Pisa (1979).

33. MacPherson R. Combinatorial differential manifolds: a symposium in honor of John Milnor's sixtieth birthday. In: Goldberg LH, Phillips A, editors. Topological ethods on Modern Mathematics. Stony Brook, NY; Houston, TX: Perish (1993). p. 203-21.

34. Anderson L, Davis JF. Mod 2 cohomology of combinatorial Grassmannians. Select Math. (2002) 8:161-200. doi: 10.1007/s00029-002-8104-4

Conflict of Interest Statement: The author declares that the research was conducted in the absence of any commercial or financial relationships that could be construed as a potential conflict of interest.

Copyright (c) 2018 Nieto. This is an open-access article distributed under the terms of the Creative Commons Attribution License (CC BY). The use, distribution or reproduction in other forums is permitted, provided the original author(s) and the copyright owner(s) are credited and that the original publication in this journal is cited, in accordance with accepted academic practice. No use, distribution or reproduction is permitted which does not comply with these terms. 